\title{
Grotesk, unpraktikabel, unsinnig, schikanös
}

Otmar Häfliger

Korrespondenz: Dr. med. Otmar Häfliger Allg. Medizin FMH i. R. Stengelmattstrasse 11 CH-6252 Dagmersellen Tel. 0627563223

otmar.haefliger@hin.ch
Im BAG Bulletin 8/10 vom 22.2.10 erläutert das Bundesamt für Gesundheit BAG «das praktische Vorgehen bei der Abrechnung der neuen Taxen und Zuschläge». Ich beschränke mich auf das ärztliche Praxislabor für Allgemeinmediziner, teilweise im Vergleich mit den anderen Labortypen, und erlaube mir (wie das BAG) die Aufführung von Beispielen aus dem Praxisalltag.

\section{Einige Beispiele aus der Praxis}

\section{Beispiel 1}

Ein Patient, 40 Jahre, erscheint morgens bei Praxisöffnung unangemeldet wegen Fiebers und Kopfschmerzen. Da ein bakterieller Infekt nicht ausgeschlossen werden kann, werden CRP $(10 \mathrm{mg} / \mathrm{l})$ und Hämatogramm3 (Lc 8,8 G/l, Granulozyten 70\%) bestimmt. In der Annahme eines viralen Infekts erhält er ein Analgetikum/Antipyretikum. Wegen deutlicher Verschlechterung mit weiterem Fieberanstieg, Kopf- und Halsschmerzen meldet sich der Patient abends erneut: $\mathrm{T}_{\mathrm{ax}} .39,8^{\circ} \mathrm{C}$, Tachypnoe $40 / \mathrm{Min}$., Tachykardie 128/Min., Rachen leicht gerötet, sonst oB. Erneut CRP (98 mg/l) und Hämatogramm3 (Lc 16 G/l, Granulozyten $75 \%$ ). Bei wahrscheinlichem bakteriellem Infekt wird ein Antibiotikum verordnet.

In der Allgemeinpraxis dürfen bei gleichem Arbeitsaufwand morgens (inkl. Blutentnahme) Fr. 32.94, abends Fr. 22.- (keine Präsenztaxe, keine Blutentnahme) verrechnet werden.

Wird der Patient resp. die Blutprobe weitergeschickt, ergeben sich im Vergleich zur Allgemeinpraxis folgende Kosten:

\begin{tabular}{|c|c|c|c|}
\hline Labortyp & morgens & abends & Total \\
\hline Allgemeinpraxis & 32.94 & 22.00 & 54.94 \\
\hline $\begin{array}{l}\text { Offizin von Apothekern }{ }^{1} \\
\text { (10 km entfernt) }\end{array}$ & 35.60 & 35.60 & 71.20 \\
\hline $\begin{array}{l}\text { Privatlabor ( } 30 \mathrm{~km} \\
\text { entfernt, Resultat } \\
\text { frühestens nach } \\
\text { Stunden) })^{2}\end{array}$ & 44.00 & 44.00 & 88.00 \\
\hline $\begin{array}{l}\text { Spitallabor C (10 km } \\
\text { entfernt, Resultat } \\
\text { frühestens nach } 1 \text { Std.) }\end{array}$ & 52.60 & 52.60 & 105.20 \\
\hline $\begin{array}{l}1 \text { führt keine Analysen a } \\
2 \text { Blutentnahme in der } P\end{array}$ & $\begin{array}{l}\text { us (warum } \\
\text { raxis, Versa }\end{array}$ & $\begin{array}{l}\text { ohl?) } \\
\text { d der Probe }\end{array}$ & \\
\hline
\end{tabular}

Das Ganze ist grotesk, vor allem auch, weil bei der Abendkonsultation in der Praxis wegen der fehlenden Verrechenbarkeit der Präsenztaxe auch die Blutentnahme und die Zuschläge Suffix C nicht verrechnet werden können. Dafür gibt es keine rationale Begründung.
Der Krankheitsfall verteuert sich zusätzlich, weil nach der auswärtigen Analyse mindestens noch eine 5-minütige zusätzliche Konsultation nötig ist.

\section{Beispiel 2}

Dieses Beispiel liefert das BAG selber im BAG Bulletin 8/10 auf Seite 162: Quick-Bestimmung bei Hausbesuch (1700.00): Präsenztaxe und Analyse (mit Suffixzuschlag) werden nur vergütet, wenn der Arzt (nicht die MPA!) anlässlich des Besuches die Blutprobe entnimmt und die Analyse («zwingend») in seiner Praxis durchführt. Das BAG verweist auf die Zulassungsbedingung in Art. 54 Abs. 1 Bst. a Ziff. 3 KVV, wonach «das Praxislaboratorium räumlich und rechtlich Teil der Praxis des behandelnden Arztes oder der behandelnden Ärztin ist».

Das Ganze ist unsinnig, da die Analyse ohne Qualitätsverlust beim Patienten zu Hause durchgeführt werden kann (Kosten Fr. 12.-). Geht die MPA zur Blutentnahme zum Patienten, «kann weder die Analyse noch die Präsenztaxe zu Lasten der Krankenversicherung abgerechnet werden». Der Allgemeinmediziner kann die Blutprobe jedoch an ein Privatlabor senden, was dann Fr. 31.- kostet. Er kann aber auch ein Privatlabor für Probenentnahme und Analyse beauftragen, wodurch die Analyse Fr. 78.60 (Entfernung 10 km) kosten würde. In beiden Fällen entstehen Zeitverzögerungen und werden zusätzliche (telefonische) Konsultationen nötig.

\section{Beispiel 3}

Der Patient wird für die Blutentnahme morgens nüchtern (Lipidstatus) aufgeboten. Da die Resultate erst 45 Minuten später zur Verfügung stehen, findet die Konsultation mit Resultat-Besprechung abends nach Arbeitsschluss statt, damit er zur Arbeit gehen kann,

Das ärztliche Praxislaboratorium darf die Analysen (und nicht nur die Präsenztaxe und Suffixzuschläge) nur verrechnen, wenn auch am Morgen eine (unnötige) Konsultation (mind. TP 17.76) stattgefunden hat.

Der Weg des BAG führt über die Einsendung an ein Privatlabor, wobei Kosten von Fr. 35.50 (ohne Blutentnahme, die ja wie auch der Probenversand durch die MPA gratis durchgeführt wird) entstehen.

Das Ganze ist unpraktikabel und verursacht auch hier (um 25\%) höhere Labor- und vor allem Arbeitskosten in Form von Arbeitsausfall für den Patienten, ohne dass die Arbeit der MPA bezahlt wird.

\section{Beispiel 4}

Der Patient wird vom Spezialisten zur CT im Spital angemeldet. Die Radiologie des Spitals beauftragt den 
Hausarzt, wegen der Kontrastmittelbelastung, mit einer vorgängigen Kreatinin-Bestimmung.

Das ärztliche Praxislaboratorium darf die Analysen (wie auch die Blutentnahme) nicht verrechnen, da laut BAG Bulletin 8/10 Seite 162 «bei Analysenaufträgen, die von einem anderen Leistungserbringer stammen, (anderer Arzt, Spital, Hebamme, Chiropraktor) kein Anspruch auf Abgeltung durch die Krankenversicherer besteht».

\section{Fazit}

Die im BAG Bulletin 8/10 dargestellte Interpretation des Praxislabors ist legalistisch. Es geht dem BAG offensichtlich weder um Kosteneinsparung noch um Qualität noch um Patientenfreundlichkeit. Die Kosten sind höher, die Qualität des Praxislabors ist seit vielen Jahren nachgewiesen, Patientennutzen und -sicherheit sind kein Thema, schon gar nicht ein sinnvoller, speditiver Sprechstunden-Ablauf. Beabsichtigt ist der

\section{Es geht dem BAG offensichtlich weder um Kosteneinsparung noch um Qualität noch um Patientenfreundlichkeit. Beabsichtigt ist der Ruin des Praxislabors}

Der Arzt muss also eine Konsultation veranstalten und die Analyse selber indizieren (Fr. 13.44 plus Konsultations-Taxe) oder die Analyse durch das Spital selbst durchführen lassen (Fr. 16.10), wofür der Patient mindestens eine Stunde früher (wenn nicht schon am Vortag) im Spital erscheinen muss.

Das Ganze ist schikanös, belastet den Patienten unnötig und verursacht auch hier wieder unnötige Kosten. Da diese den Krankenversicherer nicht betreffen, sind sie offenbar irrelevant.
Ruin des Praxislabors. Dem Hausarzt wird ein wichtiges Instrument für Diagnose und Therapiebeurteilung aus der Hand genommen, seine Tätigkeit unnötig erschwert, zum Teil verunmöglicht. Damit kommt man dem couchepinschen Ziel der Abschaffung der ärztlichen Grundversorgung wieder einen Schritt näher. Darf man hoffen, dass unter Bundesrat Burkhalter beim BAG anstelle von Rechthaberei und Diktatur wieder etwas Vernunft einkehrt?

\section{SWISS MEDICAL WEEKLY}

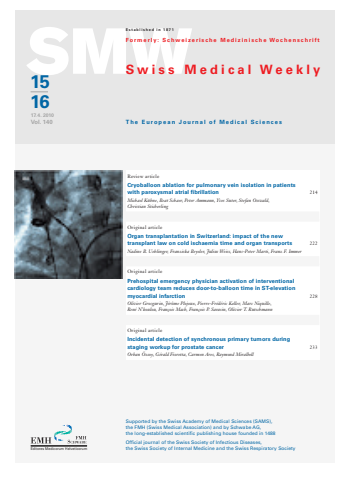

Das «Swiss Medical Weekly» ist eine international beachtete, peer reviewte Forschungszeitschrift auf Open-Access-Basis Mit Ihrem Abonnement der gedruckten Ausgabe unterstützen Sie diese insbesondere für den akademischen Nachwuchs

in der Schweiz wichtige wissenschaftliche Plattform:

Jahresabonnement (25 Ausgaben):

Fr. 150.- exkl. Versand

Bestellung per E-Mail an auslieferung@ emh.ch, im Internet unter www.smw.ch oder telefonisch unter 0614678575.

\section{Ausgabe 15/16 erscheint am 17. April 2010:}

\begin{abstract}
Cryoballoon ablation for pulmonary vein isolation in patients with paroxysmal atrial fibrillation

Michael Kühne, Beat Schaer, Peter Ammann, Yves Suter, Stefan Osswald, Christian Sticherling

Cryoballoon ablation has emerged as a novel treatment option for drug-refractory atrial fibrillation. The purpose of this manuscript is to report the initial experience of a Swiss centre performing cryoballoon ablation, and to provide a critical review of the literature.
\end{abstract}

\section{Organ transplantation in Switzerland: impact of the new transplant law on cold ischaemia time and organ transports Nadine Uehlinger, Franziska Beyeler, Hans-Peter Marti, Franz F. Immer}

The new Swiss transplant law clearly entails an increase in the frequency of organ transports. Overall CIT is not affected. However, liver transplantation is afflicted by an increase in transports and CIT. This may affect mid-term outcome and should therefore be followed closely.
Prehospital emergency physician activation of interventional cardiology team reduces door-to-balloon time in ST-elevation myocardial infarction

Olivier Grosgurin, Jérôme Plojoux, Pierre-Frédéric Keller, Marc Niquille, René N'koulou, François Mach, François P. Sarasin, Olivier T. Rutschmann

Catheterization laboratory activation by a prehospital emergency physician markedly reduces DTBT in STEMI patients.

\section{Incidental detection of synchronous primary tumours during staging workup for prostate cancer \\ Orhan Özsoy, Gérald Fioretta, Carmen Ares, Raymond Miralbell}

In patients of prostate cancer, abdomino-pelvic CT staging detects incidental second primary cancers (mostly commonly renal-cell carcinomas) with a frequency greater than that expected.

EMH Schweizerischer Ärzteverlag Editores Medicorum Helveticorum 